\title{
Establishment of organoids using residual samples from saline flushes during endoscopic ultrasound-guided fine-needle aspiration in patients with pancreatic cancer
}

\section{다 (1) $\odot(2)$}

Authors

Kenji Ikezawa*, 1, Tomoya Ekawa*, 2, Shinichiro Hasegawa ${ }^{3}$, Yugo Kai ${ }^{1}$, Ryoji Takada', Takuo Yamai ${ }^{1}$, Nobuyasu Fukutake', Hisataka Ogawa", Takashi Akazawa², Yu Mizote², Kumiko Tatsumi², Shigenori Nagata ${ }^{5}$, Kei Asukai $^{3}$, Hidenori Takahashi ${ }^{3}$ Kazuyoshi Ohkawa', Hideaki Tahara2,6

Institutions

1 Department of Hepatobiliary and Pancreatic Oncology, Osaka International Cancer Institute, Osaka, Japan

2 Department of Cancer Drug Discovery and Development, Research Center, Osaka International Cancer Institute, Osaka, Japan

3 Department of Surgery, Osaka International Cancer Institute, Osaka, Japan

4 Nitto Joint Research Department for Nucleic Acid Medicine, Research Center, Osaka International Cancer Institute, Osaka, Japan

5 Department of Diagnostic Pathology and Cytology, Osaka International Cancer Institute, Osaka, Japan

6 Project Division of Cancer Biomolecular Therapy, Institute of Medical Science, The University of Tokyo, Tokyo, Japan

submitted 15.6.2021

accepted after revision 7.10.2021

\section{Bibliography}

Endosc Int Open 2022; 10: E82-E87

DOI 10.1055/a-1713-3404

ISSN 2364-3722

(C) 2022. The Author(s).

This is an open access article published by Thieme under the terms of the Creative Commons Attribution-NonDerivative-NonCommercial License, permitting copying and reproduction so long as the original work is given appropriate credit. Contents may not be used for commercial purposes, or adapted, remixed, transformed or built upon. (https://creativecommons.org/licenses/by-nc-nd/4.0/)

Georg Thieme Verlag KG, Rüdigerstraße 14,

70469 Stuttgart, Germany

Corresponding author

Kenji Ikezawa, MD, PhD, Department of Hepatobiliary and Pancreatic Oncology, Osaka International Cancer Institute,
3-1-69 Otemae, Chuo-ku, Osaka 541-8567, Japan

Fax: +81 (6) 6945-1834

ikezawa-ke@oici.jp

\section{ABSTRACT}

Background and study aims In patients with pancreatic cancer (PC), patient-derived organoid cultures can be useful tools for personalized drug selection and preclinical evaluation of novel therapies. To establish a less invasive method of creating organoids from a patient's tumor, we examined whether PC organoids can be established using residual samples from saline flushes (RSSFs) during endoscopic ultrasound-guided fine-needle aspiration (EUS-FNA). Methods Five patients with PC who underwent EUS-FNA were enrolled in a prospective study conducted at our institution. RSSFs obtained during EUS-FNA procedures were collected. An organoid culture was considered as established when $\geq 5$ passages were successful. Organoid-derived xenografts were created using established organoids. Results EUS-FNA was performed using a 22- or 25-gauge lancet needle without complications. Patient-derived organoids were successfully established in four patients (80.0\%) with the complete medium and medium for the selection of KRAS mutants. Organoid-derived xenografts were successfully created and histologically similar to EUS-FNA samples. Conclusions Patient-derived PC organoids were successfully established using EUS-FNA RSSFs, which are produced as a byproduct of standard manipulations, but are usually not used for diagnosis. This method can be applied to all patients with PC, without additional invasive procedures, and can contribute to the development of personalized medicine and molecular research.

\footnotetext{
* These authors contributed equally.
} 


\section{Introduction}

Pancreatic cancer (PC) is one of the most aggressive malignant diseases, with a 5 -year survival rate of $9 \%$ [1]. Because it is difficult to detect PC at an early stage [2,3], the majority of patients with it are diagnosed when the disease is unresectable and they primarily undergo chemotherapy $[4,5]$. Combination chemotherapy regimens have become the standard first-line chemotherapy in patients with unresectable PC. Fluorouracil/ leucovorin plus irinotecan plus oxaliplatin (FOLFIRINOX) and gemcitabine plus nab-paclitaxel demonstrated superiority over gemcitabine monotherapy in multicenter phase III studies [4]. In patients with PC diagnosed with resectable or borderline resectable disease according to the National Comprehensive Cancer Network guidelines, neoadjuvant chemotherapy and neoadjuvant chemoradiotherapy are rapidly becoming more important. We have shown favorable treatment outcomes for neoadjuvant chemoradiotherapy followed by pancreatectomy [6-8]. Recently, neoadjuvant chemotherapy with gemcitabine plus S-1 was shown to be superior to upfront surgery in patients with resectable PC [9]. Collectively, the significance of chemotherapy is increasing regardless of resectability; however, efficient methods for drug selection in the context of personalized medicine remain to be established.

Organoids recapitulate their morphology and exhibit key features, including gene and protein expression, and cellular metabolic heterogeneity [10-12]. Recently, patient-derived organoid cultures have been demonstrated as a useful research tool for personalized drug selection and preclinical evaluation of novel therapies in patients with PC [11-14]. Tumor organoids have been generated from various sources, such as resected specimens and samples obtained from fine-needle aspiration (FNA) [12-15]. The success rate of organoid establishment ranges from $62 \%$ to $100 \%$ [10].

Endoscopic ultrasound (EUS) is primarily used to evaluate pancreatobiliary and mediastinal diseases [16-18]. EUS-guided FNA (EUS-FNA) has been established as an accurate method of tissue acquisition and is performed in a wide variety of patients with $P C$, including those who have unresectable disease [19, 20]. Tiriac et al. recently reported the successful creation of tumor organoids using aliquots of visible tissue samples obtained for pathological diagnosis by EUS-FNA in patients with pancreatic ductal adenocarcinoma [15]. Although this method is promising, serious concerns have been raised, as the amount of tissue samples obtained with EUS-FNA is limited [21,22]. If researchers use aliquots of tissue samples for organoid creation, it may negatively affect the pathological and genetic diagnosis by significantly reducing the amount of remaining samples. An additional puncture for organoid establishment may pose an increased risk of adverse events (AEs), such as bleeding and tumor seeding $[23,24]$. To establish a less invasive method of creating organoids from a patient's tumor, we examined whether PC organoids can be established using the residual samples from saline flushes (RSSFs) during EUS-FNA procedures. RSSFs are produced as a byproduct in standard manipulations to flush the inside of the FNA needle, but are usually not used for diagnosis, and have recently been used for KRAS muta- tion analysis [21]. In the present study, we examined whether PC organoids could be established using EUS-FNA RSSFs.

\section{Patients and methods \\ Study design and patients}

In a prospective study conducted at our institution, patients were enrolled from August 2020 to December 2020. Patients older than age 20 years and referred for EUS with tissue sampling of a pancreatic mass lesion suspected to be pancreatic ductal adenocarcinoma were included in the present study. Written informed consent was obtained from all patients. The study was approved by the Institutional Review Board of the Osaka International Cancer Institute and performed in accordance with the Declaration of Helsinki.

EUS-FNA was performed by or under the supervision of experienced endoscopists who were qualified by the Japan Gastroenterological Endoscopy Society. EUS-FNA was performed using a curvilinear echoendoscope (GF-UCT260; Olympus Medical Systems, Tokyo, Japan) and an ultrasound diagnostic device (ALOKA ARIETTA 850, FUJIFILM Healthcare Corporation, Tokyo, Japan). EUS-FNA was performed with a 22- and/or 25-gauge needle (EZ-Shot 3; Olympus Medical Systems; Expect Slimline, Boston Scientific Corporation, Marlborough, Massachusetts, United States). The inside of the FNA needle was flushed with $10 \mathrm{~mL}$ of saline onto a Petri dish. A slide for rapid on-site cytological evaluation (ROSE) and a slide for cytology were made with the smear method using a part of the visible sample. After confirming malignancy on ROSE, a visible sample was used for the histological diagnosis. Furthermore, RSSFs were used in this study.

The following patient data were collected: age, sex, location of the primary tumor, tumor size, needle gauge/type, needle manufacturer/brand, and the number of punctures. Pathological diagnoses were confirmed by an expert pathologist (S.N.), and staging was performed according to the UICC staging system (8th edition) [25].

\section{Tumor organoid culture}

The RSSF from several punctures was collected into one tube. The EUS-FNA RSSFs were allowed to settle under normal gravity on ice for 10 minutes, and the precipitate was collected in a tube. The precipitate was washed several times with cold PBS to remove blood cells. The washed precipitate was incubated with $2.5 \mathrm{mg} / \mathrm{mL}$ Liberase TH (MERCK; 5401135001) and $10 \mu \mathrm{g} /$ $\mathrm{mL}$ DNase I (MERCK; DN25) for 5 to 15 minutes at $37^{\circ} \mathrm{C}$ in a water bath. The digested precipitate was sheared with a $1-\mathrm{mL}$ pipette tip. In cases with a visible red pellet, erythrocytes were lysed in red blood cell lysis buffer (pluriSelect; 600005110) for 5 minutes at room temperature. Isolated cells were embedded in Matrigel (growth factor reduced, phenol red-free) (Corning, 356231 ) on ice and seeded in 24 well plates (Corning, 3738).

Tumor organoids were cultured as described in Seino et al. and Driehuis et al., with slight modifications [13,26]. Briefly, the culture medium comprised advanced DMEM/F12 (Thermo Fisher Scientific; 12634028) supplemented with $1 \times$ Glutamax (Thermo Fisher Scientific; 35050061), 10 mM HEPES (Thermo 
Fisher Scientific; 15630080), $100 \mathrm{U} / \mathrm{mL}$ penicillin/streptomycin (Thermo Fisher Scientific; 15140122), $50 \mu \mathrm{g} / \mathrm{mL}$ primocin (InviivoGen; 14860-94), 1x B27 supplement (Thermo Fisher Scientific; 17504044), $50 \mathrm{ng} / \mathrm{mL}$ epidermal growth factor (EGF; Thermo Fisher Scientific; PMG8044), $50 \mathrm{ng} / \mathrm{mL}$ fibroblast growth factor-2 (FGF2; PeproTech; 100-18B), $100 \mathrm{ng} / \mathrm{mL}$ insulin-like growth factor 1 (IGF1; BioLegend; 590904), 1.25 mM N-acetylcysteine (MERCK; A9165), 10 nM gastrin (MERCK; G9145), $1 \mu \mathrm{g} /$ mL R-spondin (PeproTech; 120-38), $5 \mu \mathrm{M}$ A83-01 (TGF- $\beta$ inhibitor) (Tocris Bioscience; 2939), $10 \mu \mathrm{M}$ Y-27632 (FUJIFILM; 259-00613), 10\% Afamin/Wnt3a CM (MBL; J-ORMW301R), and $100 \mathrm{ng} / \mathrm{mL}$ Noggin (BMP inhibitor) (PeproTech; 250-38). This medium was considered as the complete medium. Tumor organoids were cultured in parallel in three types of tumor organoid media that were designed to select functional mutations [13]. To select KRAS mutants, EGF, FGF2, and IGF1 were removed from the complete medium because KRAS mutants constitutively activated EGF signaling. SMAD4 mutants were selected with the removal of Noggin, A83-01, and Wnt3a because SMAD4 mutants suppress TGF- $\beta / B M P$ or p38MAPK signaling. In cases of TP53 mutants, $3 \mu \mathrm{M}$ Nutlin 3 (Cayman Chemical; 10004372) was added to the complete medium because TP53 mutation acquires resistance to MDM2 inhibitor Nutrin-3. Organoid cultures in which more than $30 \%$ of the organoids were larger than $200 \mu \mathrm{m}$ in diameter, or many organoids were connected to each other, were passaged. For passage, organoids were collected, washed, and disrupted by digestion with TrypLE Express (Thermo Fisher Scientific; 12604013). After passage, the organoid fragments were replated in fresh media. The organoid culture was considered as established when five or more passages were successful.

\section{Generation of organoid-derived xenografts}

Some of the established tumor organoids were suspended in a 1:1 mixture of DMEM (MERCK; D5796) and Matrigel, and 1000 organoid clusters were subcutaneously injected into the back skin of NOD.CB17-Prkdc scid/J (NOD SCID) mice (Charles River). For pathological analysis, organoid-derived xenografts were fixed in $10 \%$ formalin neutral buffer solution (FUJIFILM) before embedding and sectioning. Hematoxylin and eosin (H\&E) staining was performed on paraffin sections of the tumors. The animal procedures were approved by the Animal Experiment Committee at Osaka International Cancer Institute.

\section{Results}

\section{Patient characteristics}

The characteristics of the five patients included in the present study are summarized in - Table 1 . The median age was 68 years (range, 56 to 82 years), and three patients (60.0\%) were women. The primary tumor site was the pancreas head in three patients $(60.0 \%)$ and pancreas body/tail in two patients (40.0\%). The median size of the tumors was $30 \mathrm{~mm}$ (range, 22 to $33 \mathrm{~mm}$ ). The lancet needles used for EUS-FNA were 22- and 25 -gauge in four cases and one case, respectively. The median number of punctures was three (range, 2 to 4). Adenocarcinoma was cytopathologically proven using EUS-FNA in all cases. No complications were observed. Four patients underwent chemotherapy, including two patients who underwent neoadjuvant chemotherapy. One patient underwent upfront surgery.

- Table 1 Characteristics of study patients.

\begin{tabular}{|c|c|c|c|c|c|c|c|c|c|c|}
\hline \multirow{2}{*}{$\begin{array}{l}\text { Pa- } \\
\text { tient } \\
\text { num- } \\
\text { ber }\end{array}$} & \multirow{2}{*}{$\begin{array}{l}\text { Age, } \\
\text { years| } \\
\text { sex }\end{array}$} & \multicolumn{3}{|c|}{ EUS-FNA } & \multirow{2}{*}{$\begin{array}{l}\text { Tumor } \\
\text { loca- } \\
\text { tion }\end{array}$} & \multirow{2}{*}{$\begin{array}{l}\text { Tumor } \\
\text { size, } \\
\text { mm }\end{array}$} & \multirow{2}{*}{$\begin{array}{l}\text { Pathologi- } \\
\text { cal diag- } \\
\text { nosis }\end{array}$} & \multirow{2}{*}{$\begin{array}{l}\text { Manage- } \\
\text { ment }\end{array}$} & \multirow{2}{*}{$\begin{array}{l}\text { c/pTNM } \\
\text { classification }\end{array}$} & \multirow{2}{*}{$\begin{array}{l}\text { Stage } \\
\text { (UICC 8th) }\end{array}$} \\
\hline & & $\begin{array}{l}\text { Nee- } \\
\text { dle } \\
\text { gauge }\end{array}$ & $\begin{array}{l}\text { Manufactur- } \\
\text { er/brand }\end{array}$ & $\begin{array}{l}\text { No. } \\
\text { punc- } \\
\text { tures }\end{array}$ & & & & & & \\
\hline 1 & $74 / M$ & $22 \mathrm{G}$ & $\begin{array}{l}\text { Olympus/ } \\
\text { EZshot3 }\end{array}$ & 3 & Body & 22 & PDAC & $\begin{array}{l}\text { Chemo- } \\
\text { therapy }\end{array}$ & cT4N0M1 & pStage IV \\
\hline 2 & $68 / M$ & $22 \mathrm{G}$ & $\begin{array}{l}\text { Olympus/ } \\
\text { EZshot3 }\end{array}$ & 2 & Head & 23 & PDAC & Surgery & $\mathrm{pT} 3 \mathrm{~N} 2 \mathrm{M} 0$ & pStage III \\
\hline 3 & $82 / F$ & $25 G$ & $\begin{array}{l}\text { Olympus/EZ- } \\
\text { shot3, Boston } \\
\text { Scientific/Ex- } \\
\text { pect Slimline }\end{array}$ & 3 & Head & 33 & PDAC & $\begin{array}{l}\text { Chemo- } \\
\text { therapy } \\
\text { (NAC) }\end{array}$ & $\begin{array}{l}\text { уст2N0M0 } \\
\text { (урT2N2M0) }\end{array}$ & $\begin{array}{l}\text { ycStage IB } \\
\text { (ypStage III) }\end{array}$ \\
\hline 4 & $56 / F$ & $22 \mathrm{G}$ & $\begin{array}{l}\text { Olympus/ } \\
\text { EZshot3 }\end{array}$ & 2 & Body & 30 & PDAC & $\begin{array}{l}\text { Chemo- } \\
\text { therapy } \\
\text { (NAC) }\end{array}$ & cT2NOMO & cStage IB \\
\hline 5 & $64 / F$ & $22 \mathrm{G}$ & $\begin{array}{l}\text { Olympus/ } \\
\text { EZshot3 }\end{array}$ & 4 & Head & 30 & PDAC & $\begin{array}{l}\text { Chemo- } \\
\text { therapy }\end{array}$ & cT4N1M0 & cStage III \\
\hline
\end{tabular}

EUS-FNA, endoscopic ultrasound-guided fine-needle aspiration; M, male; F, female; PDAC, pancreatic ductal adenocarcinoma; NAC, neoadjuvant chemotherapy. 
- Table 2 Tumor organoid establishment using residual samples from saline flushes during endoscopic ultrasound-guided fine-needle aspiration.

\begin{tabular}{|c|c|c|c|}
\hline $\begin{array}{l}\text { Patient } \\
\text { number }\end{array}$ & Medium & $\begin{array}{l}\text { Establish- } \\
\text { ment of tu- } \\
\text { mor organoid }\end{array}$ & $\begin{array}{l}\text { Days to es- } \\
\text { tablished tu- } \\
\text { mor organoid }\end{array}$ \\
\hline \multirow[t]{4}{*}{1} & Complete & O & 64 \\
\hline & KRAS selection & O & 106 \\
\hline & P53 selection & - & - \\
\hline & SMAD4 selection & - & - \\
\hline \multirow[t]{4}{*}{2} & Complete & O & 54 \\
\hline & KRAS selection & O & 54 \\
\hline & P53 selection & - & - \\
\hline & SMAD4 selection & O & 63 \\
\hline \multirow[t]{4}{*}{3} & Complete & - & - \\
\hline & KRAS selection & - & - \\
\hline & P53 selection & - & - \\
\hline & SMAD4 selection & - & - \\
\hline \multirow[t]{4}{*}{4} & Complete & O & 58 \\
\hline & KRAS selection & O & 71 \\
\hline & P53 selection & - & - \\
\hline & SMAD4 selection & - & - \\
\hline \multirow[t]{4}{*}{5} & Complete & O & 84 \\
\hline & KRAS selection & O & 61 \\
\hline & P53 selection & - & - \\
\hline & SMAD4 selection & - & - \\
\hline
\end{tabular}

\section{Tumor organoid establishment and xenograft creation}

Tumor organoids were successfully established with the complete medium in four patients $(80.0 \%$ ) ( $\checkmark$ Table 2 ). In these patients, organoids were also established with the KRAS-selection medium. In Case 2, organoids were successfully established with the SMAD4-selection medium, as well as the compete medium and KRAS-selection medium ( $\bullet$ Fig. 1 ).

Finally, we examined whether we could create tumor organoid-derived xenografts. Using established organoids (Cases 1 and 2), we successfully created organoid-derived xenograft tumors. Histological examination with H\&E staining revealed carcinomas, featuring an irregular glandular structure with focal nuclear pleomorphism in Case 1 ( $\bullet$ Fig. 2a, \ Fig. 2b), and wellformed papillae with abundant intracellular mucin in Case 2 ( Fig. 2c, $\triangleright$ Fig. 2d). The histological morphology of the xenograft tumors was highly similar to that of the tissue samples actually obtained by EUS-FNA.

\section{Discussion}

Tumor organoid establishment is an exciting novel method for translational research and precision medicine. Tumor organoids can be used for patient-specific chemotherapeutic drug selection and basic scientific research on PC [11-14]. Tumor organoids have been primarily generated from surgically resected specimens, but this is not applicable to the majority of patients with $\mathrm{PC}$ who have been diagnosed with disease that is unresectable. In addition, neoadjuvant chemotherapy or chemoradiotherapy may increase the difficulty in creating tumor organoids, because neoadjuvant treatments cause shrinkage and the disappearance of PC [27-29]. Therefore, sources other than surgically resected specimens are required for the establishment of PC organoids.

In the present study, we demonstrated that tumor organoids can be successfully established using EUS-FNA RSSFs in patients with PC. RSSFs are usually considered remnant samples because the cytopathological diagnosis can be performed with visible tissue samples. A previous study reported the successful creation of PC organoids using aliquots of visible tissue samples obtained by EUS-FNA for pathological diagnosis [15]. However, an additional puncture solely for organoid establishment is undesirable, as such additional punctures may increase the risk of AEs, including tumor seeding. Yane et al. recently reported that tumor seeding occurred in $3.4 \%$ of cases [23]. Thus, the number of punctures should be reduced, especially in patients with resectable PC. In addition, using aliquots of visible tissue samples for organoid creation may negatively impact gene testing (microsatellite stability status and cancer multi-gene panel testing), which is of growing importance in treatment selection [30-32]. Park et al. reported that patients with PC with homologous recombination deficiency (approximately $20 \%$ of PC) are sensitive to platinum-containing chemotherapy [33]. Although third-generation EUS-guided fine needles have recently shown a higher histologic core procurement rate and higher success rate for genetic testing, the amount of samples is still limited [34]. The application of RSSF facilitates the creation of tumor organoids in patients with PC.

To the best of our knowledge, this is the first report of tumor organoid creation using EUS-FNA RSSFs. Tumor organoids were successfully established not only in the complete medium but also in medium for the selection of mutants, including KRAS mutations. Furthermore, we also succeeded in creating xenografts, with histological findings similar to those of the histological images of EUS-FNA. Although only a few basic research reports exist regarding the use of EUS-FNA RSSFs [21], they could be an efficient resource for tumor organoid establishment and other molecular studies.

The present study has several limitations. The number of patients enrolled in it was small. It remains unclear whether the use of EUS-FNA RSSFs significantly decreases the number of punctures in creating PC organoids. Further studies with larger sample size are required to examine the efficacy and safety of this method. However, rate of success in establishing tumor organoids was satisfactory in comparison to previous studies of tumor organoid creation [10]. Combined with the finding that 


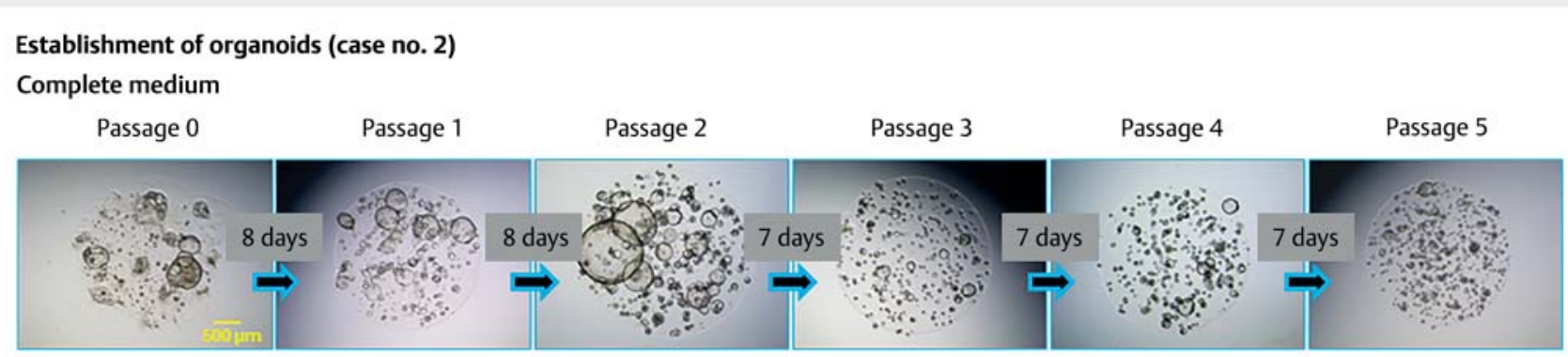

Medium for the selection of Kras mutants

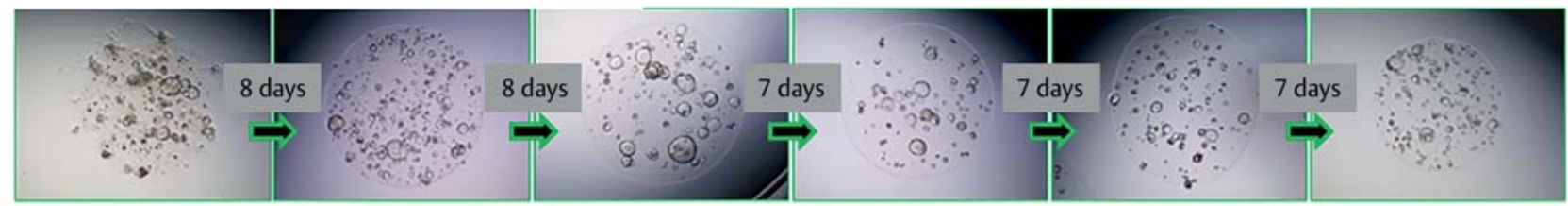

Medium for the selection of p53 mutants

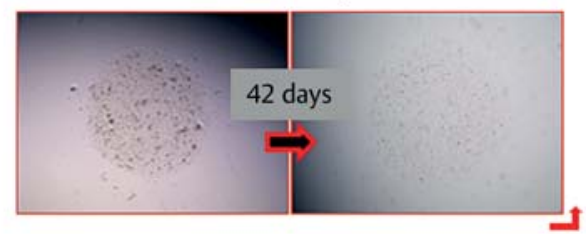

Medium for the selection of SMAD4 mutants

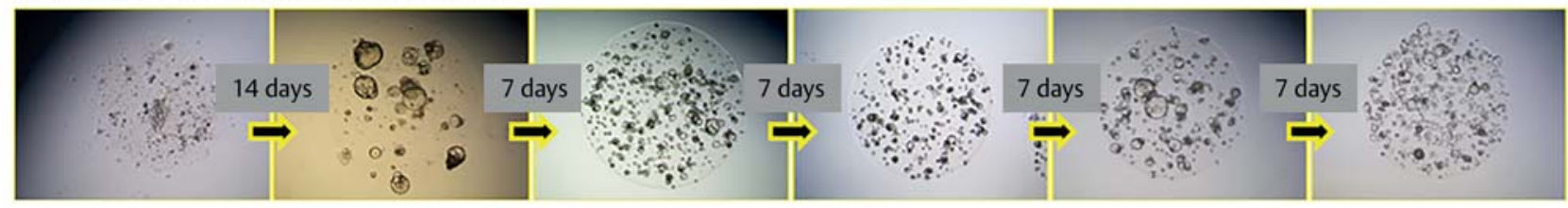

- Fig. 1 Establishment of organoids with residual samples from saline flushes (RSSFs) during endoscopic ultrasound-guided fine-needle aspiration (EUS-FNA) (Case 2).

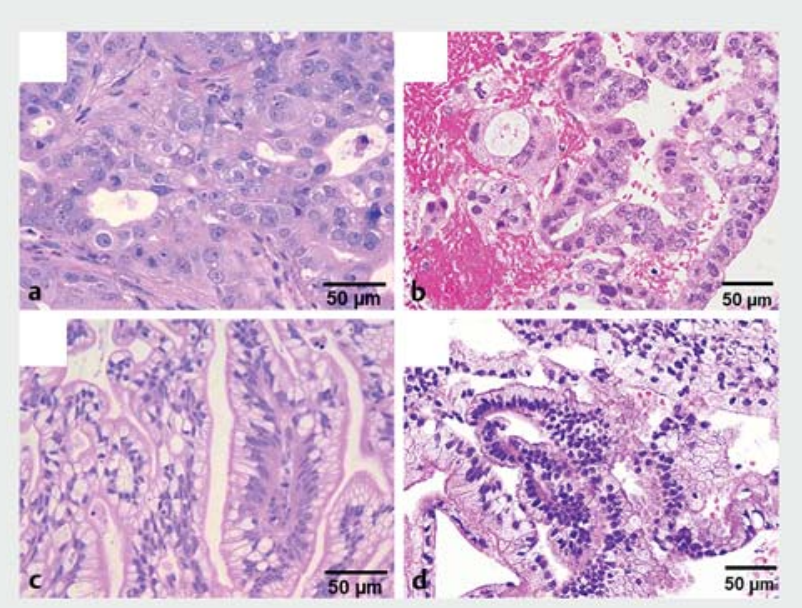

- Fig. 2 Histological examination of tumor organoid-derived xenograft and endoscopic ultrasound-guided fine-needle aspiration (EUS-FNA) (hematoxylin-eosin stain, original magnification $\times 400$ ). a Case 1 tumor organoid-derived xenograft. b Case 1 EUS-FNA. c Case 2 tumor organoid-derived xenograft. d Case 2 EUS-FNA. xenografts were successfully made by tumor organoids established by RSSFs, the results suggest that this method can be applied to further investigations of patients who undergo EUSFNA for suspected malignancy.

\section{Conclusions}

In conclusion, PC tumor organoids were successfully established using EUS-FNA RSSFs, which are usually regarded as remnant samples. This method can be applied to all patients with $P C$ without increasing $A E$, and has the potential to be a key method for personalized medicine in the future.

\section{Acknowledgements}

This work was supported by funding from Osaka International Cancer Institute.

\section{Competing interests}

\footnotetext{
The authors declare that they have no conflict of interest.
} 


\section{References}

[1] Siegel RL, Miller KD, Jemal A. Cancer statistics, 2020. CA Cancer J Clin 2020; 70: 7-30

[2] Fukuda J, Ikezawa K, Nakao M et al. Predictive factors for pancreatic cancer and its early detection using special pancreatic ultrasonography in high-risk individuals. Cancers (Basel) 2021; 13: 1-15

[3] Yoshioka T, Shigekawa M, Ikezawa K et al. Risk factors for pancreatic cancer and the necessity of long-term surveillance in patients with pancreatic cystic lesions. Pancreas 2020; 49: 552-560

[4] Franck C, Müller C, Rosania R et al. Advanced pancreatic ductal adenocarcinoma: Moving forward. Cancers (Basel) 2020; 12: 1955

[5] Ikezawa K, Kiyota R, Takada R et al. Efficacy and safety of modified fluorouracil/leucovorin plus irinotecan and oxaliplatin (mFOLFIRINOX) compared with S-1 as second-line chemotherapy in metastatic pancreatic cancer. JGH Open 2021; 5: 679-685

[6] Takahashi H, Ohigashi H, Gotoh K et al. Preoperative gemcitabinebased chemoradiation therapy for resectable and borderline resectable pancreatic cancer. Ann Surg 2013; 258: 1040-1050

[7] Takahashi H, Yamada D, Asukai K et al. Clinical implications of the serum CA19-9 level in "biological borderline resectability" and "biological downstaging" in the setting of preoperative chemoradiation therapy for pancreatic cancer. Pancreatology 2020; 20: 919-928

[8] Takahashi H, Akita H, Tomokuni A et al. Preoperative gemcitabinebased chemoradiation therapy for borderline resectable pancreatic cancer: impact of venous and arterial involvement status on surgical outcome and pattern of recurrence. Ann Surg 2016; 264: 1091-1097

[9] Unno M, Motoi F, Matsuyama Y et al. Randomized phase II/III trial of neoadjuvant chemotherapy with gemcitabine and S-1 versus upfront surgery for resectable pancreatic cancer (Prep-02/JSAP-05). J Clin Oncol 2019; 37: 189-189

[10] Frappart PO, Hofmann TG. Pancreatic ductal adenocarcinoma (Pdac) organoids: The shining light at the end of the tunnel for drug response prediction and personalized medicine. Cancers (Basel) 2020; 12: $1-18$

[11] Huang L, Holtzinger A, Jagan I et al. Ductal pancreatic cancer modeling and drug screening using human pluripotent stem cell- and patient-derived tumor organoids. Nat Med 2015; 21: 1364-1371

[12] Boj SF, Hwang CII, Baker LA et al. Organoid models of human and mouse ductal pancreatic cancer. Cell 2015; 160: 324-338

[13] Driehuis E, Van Hoeck A, Moore K et al. Pancreatic cancer organoids recapitulate disease and allow personalized drug screening. Proc Natl Acad Sci U S A 2019; 116: 26580-26590

[14] Tiriac H, Belleau P, Engle DD et al. Organoid profiling identifies common responders to chemotherapy in pancreatic cancer. Cancer Discov 2018; 8: 1112-1129

[15] Tiriac H, Bucobo JC, Tzimas D et al. Successful creation of pancreatic cancer organoids by means of EUS-guided fine-needle biopsy sampling for personalized cancer treatment. Gastrointest Endosc 2018; 87: $1474-1480$

[16] Ikezawa K, Shigekawa M, Yamai T et al. Endoscopic biliary stenting as the risk factor for cholangitis after endoscopic ultrasound in patients with biliary strictures. J Gastroenterol Hepatol 2021; 36: 1263-1266

[17] Ikezawa K, Shigekawa M, Sengoku K et al. Left atrial appendage thrombus detected by transesophageal examination with linear endoscopic ultrasound. Clin Case Rep 2019; 7: 1327-1330

[18] Iwashita T, Uemura S, Mita $\mathrm{N}$ et al. Utility of endoscopic ultrasound and endoscopic ultrasound-guided fine-needle aspiration for the di- agnosis and management of pancreatic cystic lesions: Differences between the guidelines. Dig Endosc 2020; 32: 251-262

[19] Mangiavillano B, Sosa-Valencia L, Deprez P et al. Tissue acquisition and pancreatic masses: Which needle and which acquisition technique should be used? Endosc Int Open 2020; 8: E1315-E1320

[20] Yamabe A, Irisawa A, Bhutani MS et al. Efforts to improve the diagnostic accuracy of endoscopic ultrasound-guided fine-needle aspiration for pancreatic tumors. Endosc Ultrasound 2016; 5: 225-232

[21] Matsumoto K, Kato H, Nouso K et al. Evaluation of local recurrence of pancreatic cancer by KRAS mutation analysis using washes from endoscopic ultrasound-guided fine-needle aspiration. Dig Dis Sci 2020; 65: 2907-2913

[22] Kai Y, Ikezawa K, Takada R et al. Success rate of microsatellite instability examination and complete response with pembrolizumab in biliary tract cancer. JGH Open 2021; 5: 712-716

[23] Yane K, Kuwatani M, Yoshida M et al. Non-negligible rate of needle tract seeding after endoscopic ultrasound-guided fine-needle aspiration for patients undergoing distal pancreatectomy for pancreatic cancer. Dig Endosc 2020; 32: 801-811

[24] Kanno A, Yasuda I, Irisawa A et al. Adverse events of endoscopic ultrasound-guided fine-needle aspiration for histologic diagnosis in Japanese tertiary centers: Multicenter retrospective study. Dig Endosc 2020: doi:10.1111/den.13912

[25] Brierley J, Gospodarowicz M, Wittekind C. TNM classification of malignant tumours. 8th ed. Hoboken (New Jersey), US: Wiley-Blackwell; 2017

[26] Seino T, Kawasaki S, Shimokawa M et al. Human pancreatic tumor organoids reveal loss of stem cell niche factor dependence during disease progression. Cell Stem Cell 2018; 22: 454-467.e6

[27] Yin L, Pu N, Thompson E et al. Improved assessment of response status in patients with pancreatic cancer treated with neoadjuvant therapy using somatic mutations and liquid biopsy analysis. Clin Cancer Res 2021; 27: 740-748

[28] Miyasaka Y, Ohtsuka T, Kimura R et al. Neoadjuvant chemotherapy with gemcitabine plus nab-paclitaxel for borderline resectable pancreatic cancer potentially improves survival and facilitates surgery. Ann Surg Oncol 2019; 26: 1528-1534

[29] Yoo C, Hwang I, Song T] et al. FOLFIRINOX in borderline resectable and locally advanced unresectable pancreatic adenocarcinoma. Ther Adv Med Oncol 2020: doi:10.1177/1758835920953294

[30] Pishvaian M], Blais EM, Brody JR et al. Overall survival in patients with pancreatic cancer receiving matched therapies following molecular profiling: a retrospective analysis of the Know Your Tumor registry trial. Lancet Oncol 2020; 21: 508-518

[31] Takada R, Ikezawa K, Kiyota R et al. Microsatellite instability status of pancreatic cancer and experience with pembrolizumab treatment. Suizo 2021; 36: 120-127

[32] Marabelle A, Le DT, Ascierto PA et al. Efficacy of pembrolizumab in patients with noncolorectal high microsatellite instability/ mismatch repair-deficient cancer: Results from the phase II KEYNOTE-158 study. J Clin Oncol 2020; 38: 1-10

[33] Park W, Chen J, Chou JF et al. Genomic methods identify homologous recombination deficiency in pancreas adenocarcinoma and optimize treatment selection. Clin Cancer Res 2020; 26: 3239-3247

[34] Imaoka H, Sasaki M, Hashimoto Y et al. Impact of endoscopic ultrasound-guided tissue acquisition on decision-making in precision medicine for pancreatic cancer: beyond diagnosis. Diagnostics 2021; 11: 1195 